munication with the perforations in the teeth. Further still, a special instinct leading the snake to make use of this wonderful weapon of offence, and suitable nerves to regulate its complicated action.

Now, unless all these numerous variations - and they might fairly be multiplied by subdivision-had in the first instance appeared simultaneously in one individual, and unless all had been duly connected, the whole apparatus would have been useless, and there would have been nothing of which natural selection could avail itself. Useful intermediate forms there can be none. A rifle is a more formidable weapon than a lance or dart, but of what use would be a thing half-way between the two? The venom-discharging apparatus has in it no part which could possibly be dispensed with.

To give one more instance. The tongue of the woodpecker is moved forwards in a singular way; not simply, as usual, by a muscle and sinew in front of the base of the tongue, but by a sinew terminating in a loop, through which passes another sinew from behind the tongue which, doubling through the loop, is attached to the base of the tongue. By this means, when the muscle is contracted, the tongue is drawn forward with a double velocity, which is to this bird specially useful. Now, it is impossible for any ingenuity to devise an action intermediate between this and the usual simple pull in respect of utility or complexity. But there is much more here than "a particular variation." The first woodpecker that possessed this structure must have had it in complete order, for otherwise the tongue would not move at all. In that woodpecker it must have commenced to exist in a rudimentary form before birth, in a germ possessing novel powers.

And here I must ask, How is it that anyone questions the Duke of Argyll's statement that " all organs do actually pass through rudimentary stages in which actual use is impossible"? Is it not precisely this which is implied in the Darwinian statement that "from the variable constitution of the ovum probably arises the varying structure of the organism developed from it "? What was afterwards developed was at first rudimentary, and useless. This is equally true of the whole organism-say of the serpent, or of the bird-and of the entirely novel and complicated apparatus found in them.

To call the apparatus in either serpent or bird " a particular variation" would be to give up the whole case for Darwinism. A wonderful combination of many particular variations has to be accounted for ; and, so far as I can see, Darwinism utterly fails to account for it. There are thousands of cases presenting the same difficulty.

There are simpler cases of specific change, in which the con. currence, the simultaneous appearance, of many slight and particular variations is not indispensable, but only their succession in due order in the course of many generations. Here, there is some room for the theory. Thus perhaps, possibly, we might get a giraffe. But I prefer a theory which, if true at all, accounts as readily for the most complicated apparatus as for the simplest forms of living things.

Hotel Faraglioni, Capri, January $3 \mathbf{I}$.

Probably many readers of the recent discussion on the transmission of acquired characters will regret that a mole definite conclusion has not been arrived at. This is probably due to the fact that the premises now in our possession do not admit of a definite answer yet being given. Those who assume that there is no evidence in favour of the transmission of acquired characters are mostly, I presume, supporters of "the continuity of the germ-plasm" theory of Weismann. Almost everyone admits that individuals may and do acquire certain characters due to change in environment, use, disuse, \&c. ; but while many maintain that these characters are transmitted to offspring, others deny that such is the case, or think that the evidence is in. sufficient. In supporting "the continuity of the germ-plasm" theory it is impossible to suppose that the germ-plasm is continued from one generation to another like a portion of entailed property. For each individual gives off thousands of ova or spermatozoa as the case may be, only a very few of which go to produce new individuals; therefore there is a dissipation of "germ-plasm,"-that is to say, in the germinal cells of mammals of to-day there cannot be any of the identical "germplasm" which existed in their remote invertebrate ancestors ages ago. For all this dissipation there must be some construc. tive process, otherwise the germ-plasm would come to an end.
From whence is derived this constructive material? Clearly from the exterior, for a fertilized ovum obtains material from without to admit of growth and elaboration. The constructive material, then, which the "germ-plasm" obtains-to admit of its liberal dissemination each generation-is derived from the external world, via the organism with which it is incorporated, or indeed of which it forms a part. Seeing, then, that the organism-from which this germinal matter is derived-can acquire characters - that is, undergo certain definite changes in response to altered conditions-then it seems reasonable to suppose that that part of it which ultimately finds its way to the germ-cells, is also modified during its transmission, and will therefore have more or less effect upon the forthcoming generation. But how much variation is due to the above cause, and how much to the almost infinitely various possible combinations of the two unlike germinal elements, it is impossible to say.

J. COWPER.

\section{Easy Lecture Experiment in Electric Resonance.}

AN experiment, exhibited by me in its early stages at the Royal Institution a year ago, and since shown here in various forms, on the overflow of one Leyden jar by the impulses accumulated from a similar jar discharging in its neighbourhood, is so simple an illustration of electric resonance, and so easily repeated by anyone, that I write to describe it.

Two similar Leyden jars are joined up to similar fairly large loops of wire, one of the circuits having a spark-gap with knobs included, the other being completely metallic, but of an adjustable length.

The jar of this latter circuit has also a strip of tinfoil pasted over its lip so as to provide an overflow path complete with the exception of an air-chink, $c$. It is important that this overflow path be practically devoid of self-induction. A jar already perforated could be well utilized for the purpose.

Then if the two circuits face each other at a reasonable distance, and if the slider, $s$, is properly adjusted, every discharge of A causes B to overflow. A slight shift of the slider puts them out of tune.
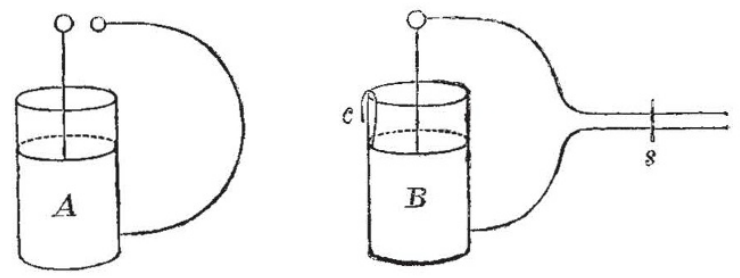

Instead of thus adjusting by variable self-induction, my assistant, Mr. Robinson, has made a slight modification by using a condenser of variable capacity, consisting of two glass tubes coated with tinfoil, one sliding into the other, and joined by a flexible loop of wire; an easy overflow from one coat to the other being likewise provided. On making this loop face the discharging circuit of an ordinary Voss machine with customary small jars in situ, bright sparks at the overflow gap occur whenever the common machine sparks are taken, provided the sliding condenser be adjusted to the right capacity by trial.

There is little or no advantage in using long primary sparks; the vibrations are steadier and more definite with short ones. It is needless to point out that the 2 jars constitute respectively a Hertz oscillator and receiver, but fair precision of timing is more needed with these large capacities than with mere spheres or discs, because the radiation lasts longer and there are more impulses to accumulate. Hence actual resonance as distinguished from the effect of a violent solitary wave is better marked. Moreover, the sparks are bright enough to be easily seen by a large audience.

University College, Liverpool.

\section{African Monkeys in the West Indies.}

WITH reference to the note in NATURE of February 13 (p. 349 ), on the occurrence of an Old-World monkey in Barbados, I may point out that the same West African monkey (Cercopithecus callitrichus) has also been introduced and is now found wild in St. Kitts (cf. Sclater, P.Z.S., 1866, p. 79). It likewise 